\title{
A Comparative Study of Data Collection Routing Protocols for Water Meter Reading Devices Network
}

\author{
Vu Chien Thang \\ Faculty of Electronics and Communications Technology, University of Information and Communications Technology, \\ Thai Nguyen, 250000, Viet Nam \\ Email: vcthang@ictu.edu.vn
}

Received: 26 August 2020; Accepted: 28 October 2020; Published: 08 December 2020

\begin{abstract}
This paper presents a comparative study of data collection routing protocols for the network of water meter reading devices. Water meters added with smart reading devices that could read the amount of water consumption and send it to the server via radio. Smart reading devices use a limited battery power source, therefore, it is necessary to employ energy-efficient data collection routing protocols to the network of water meter reading devices to increase network lifetime. CTP and RPL are two data collection protocols, which focused on research and evaluation in this paper. Simulation results in different channel quality conditions show that the RPL protocol achieves better energy efficiency than the CTP-0 and CTP-31 protocols. Although the successful data delivery ratio of RPL protocol is lower than that of CTP-31 protocol, data collected periodically in the network, so the loss of several data packets is acceptable.
\end{abstract}

Index Terms: Routing protocol for smart water meters, automatic meter reading, collection tree protocol, RPL protocol, network performance evaluation.

\section{Introduction}

Currently, the manual water meter reading is still widely used in Vietnam. This manual method is a waste of time and human labor. Thus, it is necessary to have a more suitable solution to replace current solutions, which have been used for a long time, to overcome existing limitations such as leak, wastefulness, bulky operation, inadequate accuracy, lack of consistency and low technology application. To solve this problem, water meters added with smart reading devices that could read the amount of water consumption and send it to the server via radio. The water meter reading device uses optical character recognition technology to transfer photos of the needed indicators into numbers and send them to the gateway via radio wave, from there continuously forwarding data to the server.

Smart reading devices use a limited battery power source, therefore, it is necessary to employ energy-efficient data collection routing protocols to the network of smart reading devices to increase network lifetime. In this paper, researching and evaluating protocols which collect data for the network of water meter reading devices according to the IEEE 802.15.4 standard will be focused on.

The remaining content of the paper is constructed as follows. Firstly, some related studies will be introduced. Next, several simulation and performance evaluation results of data collection routing protocols for smart water meter reading devices are presented. Finally, conclusion and future work of this research will be given.

\section{Related Works}

Currently, the solution to AMR (Automatic Meter Reading) has been researched and applied in many countries around the world. The infrastructure of AMR is defined to include components such as hardware, communication software, transmission systems, and data management software. These components are connected to form a network between measurement devices and business utility systems that allow data collection and distribution to customers and participants. Data from measurement devices is sent to the Meter Data Management System (MDMS) [1]. The MDMS system is responsible for stored-data managing and data analyzing in order to provide useful information for customer's utilities.

Figure 1 illustrates the contributing components of automatic measurement infrastructure. In the automatic measurement infrastructure, water, electricity, and gas measuring devices are deployed at households and sent to the server via information transmission networks. 


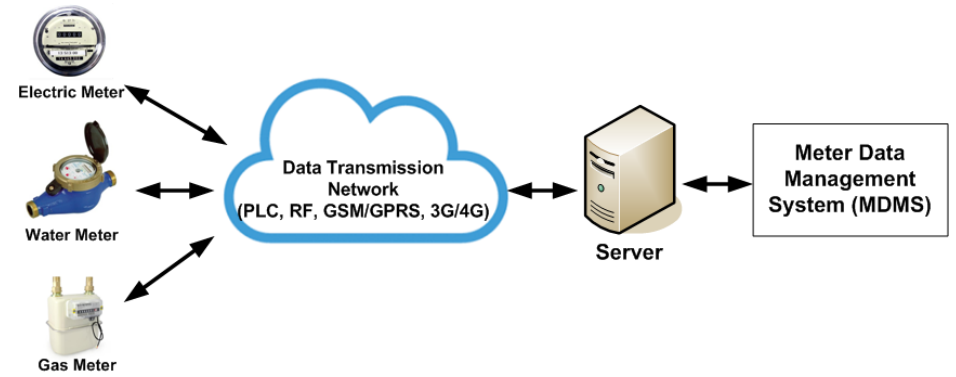

Fig. 1. Components of the automatic measurement infrastructure

In [2], the authors introduced the overview of automatic meter reading system, starting from discussing potential advantages to introducing the development process of the automatic meter reading system. The authors also launched four main types of AMR communication network, including transmission network on power conveyance line (PLC), the mobile cellular network, internet network, and short-distance radio networks such as WiFi, Bluetooth, Zigbee/IEEE 802.15.4. Nowadays, wireless communication technologies are widely used with merits such as low cost and easy deployment. In addition, the structure of the wireless AMR system is simpler and saves on labor cost more than the wired AMR system.

In [3], the authors have presented a sample product for a solution of smart water measurement. Measuring devices have been designed to operate at $169 \mathrm{MHz}$ radio frequency. Smart water meters measure the amount of water consumption through the Hall effect sensor and send the measured data to a gateway connected to the GSM/GPRS network.

In [4], the authors proposed an automated system for measuring power consumption based on a combination of GSM/GPRS and Zigbee/IEEE 802.15.4 communication technologies. In this system, the communication modules Zigbee/IEEE 802.15.4 are mounted on the meters to send measured data to the collection point.

Broadband over Power-Line (BPL) [5], [6] is another typical medium for data communication over power lines. The BPL-based system has the following advantage: no new wire, covering a wide range of convenient connections, the reliability of access and high speed. However, BPL-based system is an expensive system because it requires other components such as the collector, concentrator and master station.

In [7], the author proposed a solution for water factories in Vietnam using automatic meter reading technology. With this solution, customers and water suppliers can monitor the amount of water consumption, water quality parameters from anywhere via the internet.

In [8], the authors presented a system which reads data from water meters based on Zigbee/IEEE 802.15.4 technology. This system consists of 2 main parts. The under network includes smart water meters, data collectors and data concentrators. The upper network includes computers and servers to manage information, display data and print reports. In [8], authors have focused on studying the star topology and smart water meters that directly send data to the collectors. The star network approach is simple and useful, but it constrains the range of the smart water meter network to that of the physical transmission range of the radio transceivers.

In this paper, the model of the automatic water meter reading devices network illustrated in figure 2 . Water meter reading device connects with other devices and sends data to the gateway via the IEEE 802.15.4 standard.

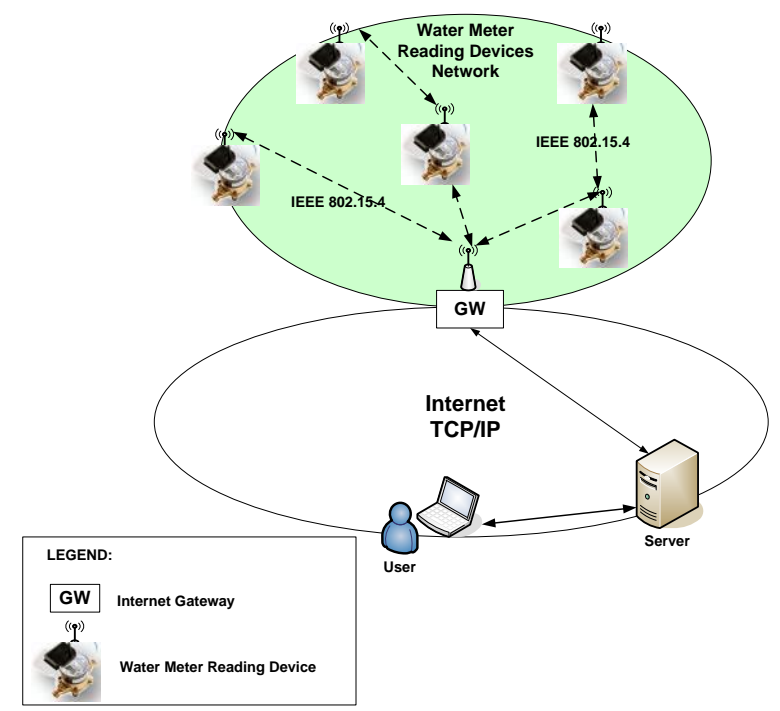

Fig. 2. Model of the automatic water meter reading devices network 
The network of water meter reading devices operates based on the data collection protocol in order to gather data from water meter reading devices. In this paper, data collection routing protocols, including Collection Tree Protocol (CTP) and IPv6 Routing Protocol for Low-power and lossy networks (RPL) will be focused on to evaluate based on the tree topology.

The CTP protocol [9] allows delivering a data packet to a collection point (called the root node) by using a minimum-cost routing tree. The cost is often calculated as the number of expected transmissions (ETX) [10] in such a way that the number of transmissions from nodes sending data on the route to the root node is the least.

The RPL protocol $[12,13]$ is designed for low-power and lossy networks with nodes that are limited in resources and interconnected by lossy links (packet loss). RPL is a distance vector protocol. This protocol builds a network structure consisting of one or more DODAG (Destination Oriented Direct Acyclic Graph). The routes are constructed from nodes in the network to one of the root nodes of the DODAG.

Currently, CTP and RPL protocols use ETX as its routing metric to avoid lossy links [14]. ETX is the inverse of the product of the forward delivery ratio, $\mathrm{D}_{\mathrm{f}}$ and the backward delivery ratio, $\mathrm{D}_{\mathrm{b}}$, which takes into account link asymmetry.

$$
\operatorname{ETX}=\frac{1}{D_{f} \cdot D_{b}}
$$

$D_{b}$ refers to the packet reception ratio, while $D_{f}$ refers to the acknowledgment reception ratio. Routing protocols based on the ETX metric provide high-throughput routes on multi-hop wireless networks since ETX minimizes the expected total number of packet transmissions required to successfully deliver a packet to the destination. This will result in a minimum energy path.

\section{Performance Evaluation of Data Collection Routing Protocol for Water Meter Reading Devices Network}

\subsection{The Scenario of Evaluation}

Figure 3 describes the network topology model considered in this paper. The network is divided into many different small clusters. Since all the clusters are the same, this research only simulates and evaluates data collection routing protocols in one cluster. The network topology in one cluster is considered as a graph. In the graph of modeling theory, the connectivity of a water meter reading devices network is described by a graph $G=(V, E)$, where $V$ (vertices) is the set of water meter reading devices, and $\mathrm{E}$ (edges) describes the adjacency relation between devices.

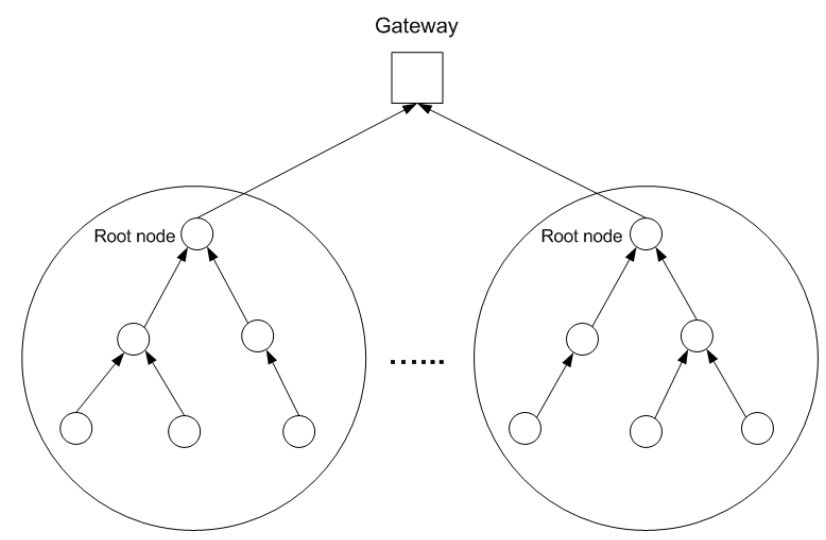

Fig. 3. The network topology is subdivided into different small clusters

The connection model used to evaluate the simulation in this paper is the UDI model [15]. Figure 4 describes the UDI model considered in this paper. In the UDI model, nodes are situated arbitrarily in the plane. Two nodes could communicate directly with each other only if their Euclidean distance is at most 1, and if the receiver is not disturbed by a third node with Euclidean distance less than or equal to a constant $\mathrm{R} \geq 1$. 


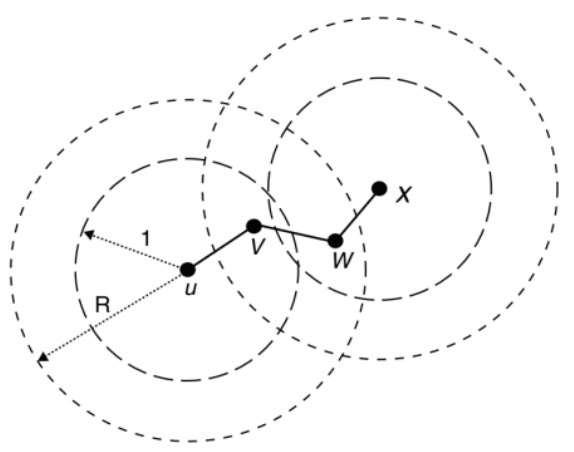

Fig. 4. UDI model

The assumptions set for the simulation problem are: nodes are heterogeneous and there are two types of nodes, sensor nodes, and root nodes; the sensor nodes send data to the root nodes via other intermediate sensor nodes; root nodes collect data from sensor nodes and directly send data to collection points; during the entire operation of the network, nodes transmit the same unchanged power level; no data collection is made in the network; all data collected by the root nodes are sent to the collection point; the sensor nodes are fixed, the network is considered static.

The network performance was evaluated by using Cooja simulator [16]. Simulated network in a cluster consists of 37 nodes placed in a grid area of 100mx100m. These nodes are distributed the same as in a small area of the urban area. Each cluster consists of a root node placed in the cluster center (node number 37) and another 36 nodes are water meter reading devices that are responsible to collect and send data from the water meter to the root node, as shown in figure 5.

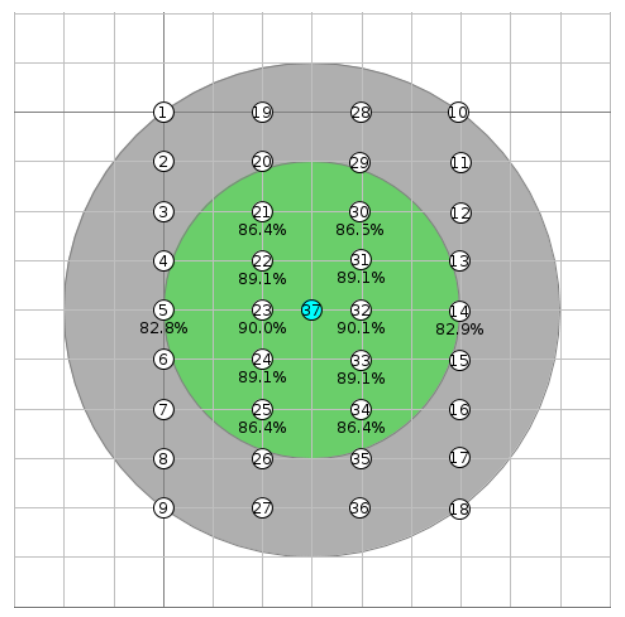

Fig. 5. The cluster model consists of 37 nodes

A normal operating state of the network, with RPL and CTP protocols used in different conditions of link quality corresponding to different successful delivery ratio, will be simulated in order to compare the network performance, the advantages and disadvantages of each protocol could be exposed. The RPL network uses UDP protocol at the transport layer. UDP is an unreliable transfer protocol, without confirmation and retransmission. For the CTP network, the author evaluated it in two different conditions: reliable transmission with the maximum number of retransmissions between hops is 31 (CTP-31) and unreliable transmission with the maximum retransmissions between hops is 0 (CTP-0). Under normal network operating conditions, each water meter reading device would send data packets to the root node with a random frequency of 1 packet/1 minutes.

Table 1. Simulation Scenario

\begin{tabular}{ll}
\hline Parameter & Value \\
\hline Radio model & UDI (Unit Disk Graph with Distance Interference) \\
Number of nodes & 37 \\
Network size $(\mathrm{m} \times \mathrm{m})$ & $100 \times 100$ \\
Communication range of node & Transmission range: $30 \mathrm{~m}$, Interference range: $50 \mathrm{~m}$ \\
Successful packet reception and transmission ratio $(\%)$ & $85,91,97$ \\
Routing protocol & RPL, CTP-0, CTP-31 \\
Data packet interval & $60 \mathrm{~s}$ \\
Data packet initialization & All nodes except the root node \\
MAC protocol & CSMA/ContikiMAC \\
\hline
\end{tabular}


The parameters used in the simulation are summarized in Table 1. Radio communication model used in the simulation is the UDI model, in which the effective transmission range is 30 meters and the interference range is 50 meters. The energy-saving protocol in the MAC layer employed in the simulation scenario is ContikiMAC protocol [11, 17].

\subsection{Evaluation Metrics}

The performance of CTP and RPL protocols will be evaluated and compared through some of the following evaluation metrics.

Data delivery ratio: The first metric is the data delivery ratio (DDR). We define DDR as the ratio between the number of data packets received at the root and the total number of sent data packets by all nodes in the whole network.

$$
D D R(\%)=\frac{N_{\text {received }}}{N_{\text {data }}} .100 \%
$$

In (2), $N_{\text {received }}$ is the number of data packets received at the root; $N_{\text {data }}$ is the number of data packets sent by all nodes in the network. The higher the DDR is, the better the communication efficiency of the network becomes. Clearly, DDR equals to 1 indicates that the network can deliver all the data to the root node.

Average energy consumption: In this paper, CTP and RPL protocols have been evaluated based on the simulations. Tmote Sky hardware platform [18] built on Cooja simulation tool was used. The Tmote Sky hardware platform is equipped with a radio transceiver according to IEEE 802.15.4 standard, the same as smart reading devices. To estimate energy consumption of the Tmote Sky hardware platform, the software-based online energy estimation was used. The total energy consumption of the node is defined as [19]:

$$
E_{\text {consumption }}=U\left(I_{a} t_{a}+I_{l} t_{l}+I_{t} t_{t}+I_{r} t_{r}+\sum_{i} I_{c i} t_{c i}\right)
$$

Where $U$ is the supply voltage, $I_{a}$ is the consumption current of the microcontroller when running, $t_{a}$ is the time in which the microcontroller has been running, $I_{l}$ and $t_{l}$ are the consumption current and the time of microcontroller in low power mode, $I_{t}$ and $t_{t}$ are the consumption current and the time of the communication device in transmit mode, $I_{r}$ and $t_{r}$ are the consumption current and the time of communication device in receive mode, $I_{c i}$ and $t_{c i}$ are the consumption and the time of other components such as sensors and LEDs...

Table 2 shows the energy model of Tmote Sky, where the consumption currents are from chip manufacturer data sheets. In the energy model of Tmote Sky, the author only considers the main energy consumptions that are the radio transceiver, the microcontroller, and other small energy consumptions ignored.

Table 2. Energy Model of Tmote Sky

\begin{tabular}{lll}
\hline Component & State & Current \\
\hline \multirow{2}{*}{ MSP430 F1611 } & Active & $1,95 \mathrm{~mA}$ \\
& Low power mode & $0,0026 \mathrm{~mA}$ \\
CC2420 & Transmit $(0 \mathrm{dBm})$ & $17,4 \mathrm{~mA}$ \\
& Listen & $19.7 \mathrm{~mA}$ \\
\hline
\end{tabular}

Average number of times to change parent node: The average number of parent node change is determined based on the statistics of the number of parent node changes for each node. The network of water meter reading devices according to IEEE 802.15.4 standard operates on lossy radio links. The radio links are often unstable quality and change frequently over time. Therefore, network topology also needs changing in order to adapt to the radio communication environment. To evaluate this adaptive change, the author relies on statistics of the average number of parent node changes in the whole network.

Average hop count in the network: The hop count refers to the number of intermediate nodes through which data must pass between source and destination. Hop count is a rough measure of distance between two nodes.

\subsection{Simulation Results}

Post-simulation data is extracted, analyzed and graphed to make comparisons. Figures 6, 7, 8, and 9 below, correspond to the simulation results, compare 4 categories such as the successful data packet delivery ratio, the average power consumption, the average number of parent node changes and the average number of hops in the network with CTP-0, CTP-31, and RPL protocols.

As seen in figure 6, the network operated with the CTP-31 protocol achieves the highest efficiency in successful data packets delivery ratio. To obtain this result, the maximum number of retransmissions between hops have been set to 31 retransmissions. By using the reliable transmission mechanism, CTP-31 protocol achieved a much higher efficiency than CTP-0 protocol. Comparing with the simulation results in figure 7, CTP-31 protocol also has been 
realized that it consumes higher average power than CTP-0 protocol. This is perfectly appropriate because retransmission is an energy-consuming operation. Figure 6 also shows that the network operated with the RPL protocol receives higher efficiency of successful data packet delivery ratio than CTP-0 protocol but lower than CTP-31 protocol. The RPL network uses the UDP protocol at the transport layer. The UDP protocol provides the best effort in data packet delivery service. Such network performs the best possible in data packet delivery, but it does not guarantee that data packets are successfully delivered to the destination. The UDP protocol does not have the acknowledgment and retransmission mechanisms.

Figure 8 depicts that the network operated with the RPL protocol has higher network stability than the CTP-0 and CTP-31 protocols. This has been expressed by the much lower average number of parent node changes in the network of the RPL protocol than that of the CTP-0 and CTP-31 protocols. Thus, the RPL protocol reacts more slowly with the link quality changes in the network. Because, the network of water meter reading devices according to the IEEE 802.15.4 standard works on the channel which is lossy, low power and frequently and continuously changing in the link quality over time, this network is not necessary to respond too quickly to such changes. Therefore, the RPL protocol achieves better the stability network than the CTP-0 and CTP-31 protocols. This stability also contributes to the higher the successful data packet delivery ratio of the RPL protocol than that of the CTP-0 protocol. However, due to no acknowledgment and retransmission mechanisms, the network operated with RPL protocol has a lower successful data packet delivery ratio than the network operated with the CTP-31 protocol. The CTP-0 and CTP-31 protocols react quickly to link quality changes, reflected by the much higher average number of parent node changes in the network than that of the RPL protocol. Through simulation results, it could be said that the rapid change in the lossy and low power network topology is not really necessary. This even could reduce the successful data packet delivery ratio and generate additional energy costs when sending control messages to update the network topology.

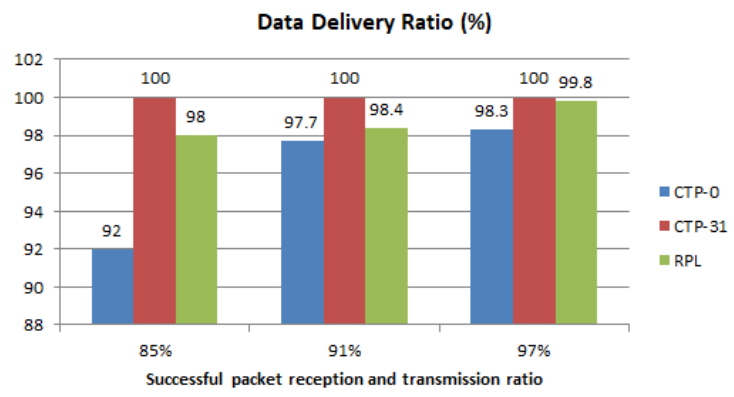

Fig. 6. Comparison in terms of successful data packets delivery ratio

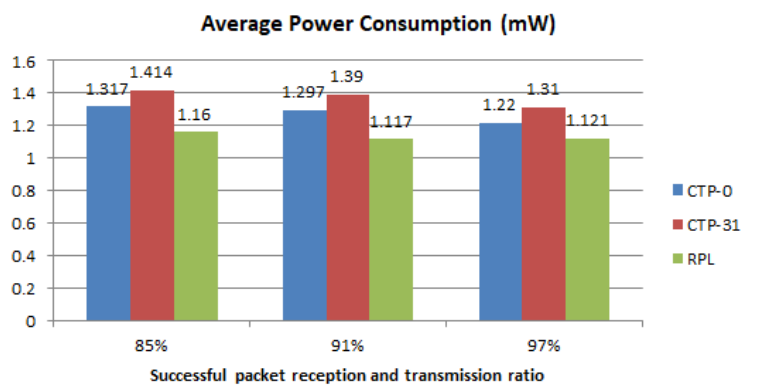

Fig. 7. Comparison in terms of average power consumption in the network

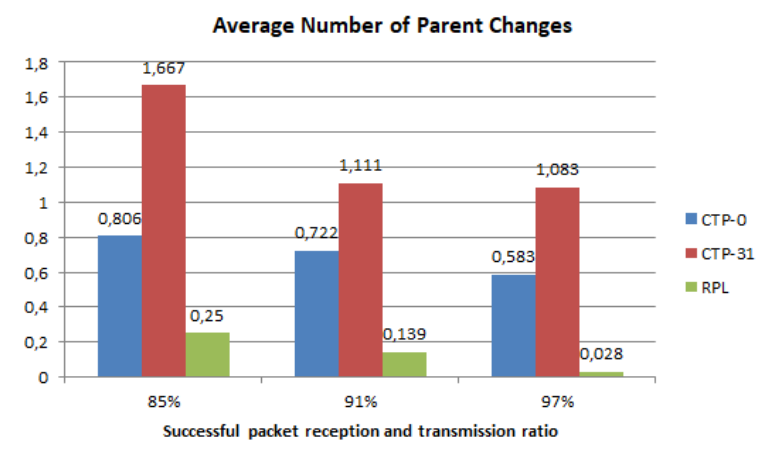

Fig. 8. Comparison in terms of average number of parent changes 


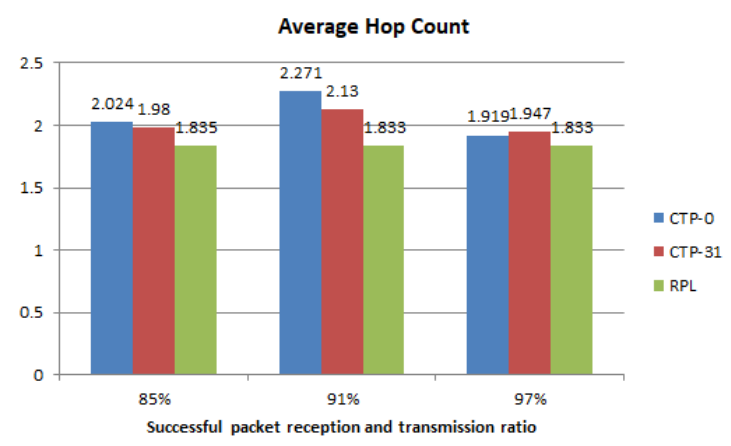

Fig. 9. Comparison in terms of average hop count

Simulation results in figure 8 illustrate that the network operated with the CTP-31 protocol has a higher average number of parent node changes than the network operated with the CTP-0 protocol. As can be seen at analyzing the operation of the CTP protocol, every time the data was transmitted/received, the link quality between the nodes would be updated and the nodes would also update the corresponding parent nodes. The network operated with CTP-31 protocol has a higher number of transmissions than the network operated with CTP-0 protocol, so the network topology is also had more times to update and adjust.

Figure 9 depicts that the network operated with the RPL protocol has a lower average number of hops than the CTP protocol. As a result, the number of times data packets must be forwarded in the RPL network is also lower than that of the CTP protocol. The average hop count is related to the communication delay in the network.

The series of simulation results show that the RPL protocol is more suitable for water meter reading devices network. The RPL protocol achieves better energy efficiency than the CTP protocol. This is appropriate for the batteryoperated network of water meter reading devices. Although the successful data delivery ratio of RPL protocol is lower than that of CTP-31 protocol, data collected periodically in the network, so the loss of several data packets is acceptable.

\section{Conclusion and Future Work}

In this paper, some evaluating and comparing performance results of data collection routing protocols RPL, CTP-0, and CTP-31 for the network of water meter reading devices have been provided. The simulation results show that the RPL protocol obtains better energy efficiency than the CTP-0 and CTP-31 protocols. The RPL protocol also achieves a relatively high successful data delivery ratio (>97\%), better than the CTP-0 protocol. In the water meter reading devices network, data collected periodically, so the loss of several data packets is acceptable. Thus, the RPL protocol is suitable for the network of water meter reading devices operated with limited battery power.

Next time, an experiment of water meter reading devices network will be deployed and evaluated in a residential area. These simulation results are useful for experimental setup in future work.

\section{References}

[1] Omowunmi M. Longe, Khmaies Ouahada, Hendrick C. Ferreira, Suvendi Rimer, "Wireless Sensor Networks and Advanced Metering Infrastructure Deployment in Smart Grid," Proceedings of International Conference on e-Infrastructure and eServices for Developing Countries, pp. 167-171, 2013.

[2] Tarek Khalifa, Kshirasagar Naik, Amiya Nayak, "A Survey of Communication Protocols for Automatic Meter Reading Applications," in IEEE Communications Surveys \& Tutorials, vol.13, no.2, pp.168-182, 2011.

[3] Gabrielli, Pizzichini, Spinsante, Squartini, Gavazzi, "Smart water grids for smart cities: A sustainable prototype demonstrator," Proceedings of 2014 European Conference on Networks and Communications, pp.1-5, 2014.

[4] Aryo Handoko Primicanta, Mohd Yunus, and Mohammad Awan, "Hybrid Automatic Meter Reading System," Proceedings of the IEEE International Conference on Computer Technology and Development, vol.2, pp. 264-267, 2009.

[5] Qingyang Liu, Bingzhen Zhao, Yirong Wang, Jing Hu, "Experience of AMR systems based on BPL in China," Proceedings of the IEEE International Symposium on Power Line Communications and Its Applications, pp. 280-284, 2009.

[6] Mingfu Li and Hung-Ju Lin, "Design and Implementation of Smart Home Control Systems Based on Wireless Sensor Networks and Power Line Communications," IEEE Transactions on Industrial Electronics, pp. 4430-4442, 2014.

[7] Vu Chien Thang, "A Solution for Water Factories in Vietnam using Automatic Meter Reading Technology," International Journal of Computer Network and Information Security (IJCNIS), Vol. 10, No. 8, Aug. 2018.

[8] Baoding Zhang, Jialei Liu, "A Kind of Design Schema of Wireless Smart Water Meter Reading System Based on Zigbee Technology," 2010 International Conference on E-Product E-Service and E-Entertainment, pp. 98-100.

[9] Omprakash Gnawali, Rodrigo Fonseca, Kyle Jamieson, Maria KazandJieva, David Moss, Philip Levis, "CTP: An Efficient, Robust, and Reliable Collection Tree Protocol for Wireless Sensor Networks," ACM Transactions on Sensor Networks, Volume 10 Issue 1, November 2013.

[10] De Couto D, Aguayo D, Bicket J, Morris R, “A high-throughput path metric for multi-hop wireless routing," In Proceedings of the 9th Annual International Conference on Mobile Computing and Networking, New York, 2003. 
[11] A. Dunkels, B. Grönvall, and T. Voigt, "Contiki - a lightweight and flexible operating system for tiny networked sensors," in Proceedings of EmNets, pp. 455-462, 2004.

[12] https://tools.ietf.org/html/rfc6550.

[13] N. Tsiftes, J. Eriksson, and A. Dunkels, "Low-Power Wireless IPv6 Routing with ContikiRPL," in Proceedings of the International Conference on Information Processing in Sensor Networks (ACM/IEEE IPSN), Stockholm, Sweden, 2010.

[14] $\mathrm{Vu}$ Chien Thang, Nguyen Van Tao, "A Performance Evaluation of Improved IPv6 Routing Protocol for Wireless Sensor Networks," International Journal of Intelligent Systems and Applications (IJISA), Vol. 8, No. 12, Dec. 2016.

[15] Azzedine Boukerche, "Algorithms and Protocols for Wireless Sensor Networks," John Wiley \& Sons Inc., ISBN: 9780470396360, 2008.

[16] Fredrik Österlind, Adam Dunkels, Joakim Eriksson, Niclas Finne, and Thiemo Voigt, "Cross-level sensor network simulation with cooja," in Proceedings of the First IEEE International Workshop on Practical Issues in Building Sensor Network Applications (SenseApp 2006), Tampa, Florida, USA, pp. 641-648, November 2006.

[17] A. Dunkels, “The ContikiMAC Radio Duty Cycling Protocol," SICS technical report, December 2011.

[18] https://insense.cs.st-andrews.ac.uk/files/2013/04/tmote-sky-datasheet.pdf.

[19] Adam Dunkels, Fredrik Osterlind, Nicolas Tsiftes, Zhitao He, "Software-based Online Energy Estimation for Sensor Nodes," Proceedings of the 4th workshop on Embedded networked sensors, 2007.

\section{Author's Profile}

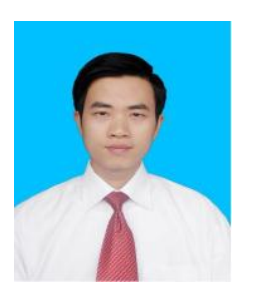

Vu Chien Thang received the B.S., M.Sc. degree in Electronics and Communications Technology from Hanoi University of Science and Technology in 2006, 2009 and Ph.D. in Telecommunications Engineering from Vietnam Research Institute of Electronics, Informatics, and Automation in 2015. He is currently a lecturer at Thai Nguyen University of Information and Communications Technology. He has published over 20 journal articles. His research interests include wireless communications networks, internet of things, embedded systems.

How to cite this paper: Vu Chien Thang, " A Comparative Study of Data Collection Routing Protocols for Water Meter Reading Devices Network", International Journal of Wireless and Microwave Technologies(IJWMT), Vol.10, No.6, pp. 1-8, 2020.DOI: 10.5815/ijwmt.2020.06.01 